\begin{tabular}{|c|c|c|c|c|c|}
\hline MUNIBE Antropologia-Arkeologia & $n^{0} 71$ & $151-161$ & DONOSTIA & 2020 & ISSN 1132-2217 • eISSN 2172-4555 \\
\hline
\end{tabular}

\title{
Pizarras numerales de época posromana y contextos arqueológicos: el yacimiento de El Castillón (Santa Eulalia de Tábara, Zamora)
}

\author{
Post-Roman numerical slates and archaeological contexts: \\ the site of El Castillón (Santa Eulalia de Tábara, Zamora)
}

PALABRAS CLAVES: Pizarras numerales. Contextos arqueológicos. Asentamientos rurales fortificados. Alta Edad Media. Meseta del Duero. GAKO-HITZAK: Arbel numeralak. Testuinguru arkeologikoak. Gotortutako landa-kokaguneak. Goi Erdi Aroa. Duero ibaiaren goi-lautada. KEY WORDS: Numerical slates. Archaeological contexts. Fortified rural settlements. Early Middle Ages. Duero's Plateau.

Iñaki MARTíN VISO(1), José Carlos SASTRE BLANCO(2), Raúl CATALÁN RAMOS ${ }^{(2)}$, Patricia FUENTES MELGAR ${ }^{(2)}$

\section{RESUMEN}

Se presenta el hallazgo de fragmentos de pizarras numerales procedentes del sitio de El Castillón (Santa Eulalia de Tábara, Zamora). El lugar es un asentamiento rural fortificado con una ocupación de los siglos V-VI, sobre el que se construyó una iglesia en los siglos IX-X. Las pizarras proceden de la intervención en la iglesia, aunque posiblemente deban relacionarse con la ocupación posromana. En el artículo, se estudian esas pizarras numerales y se analizan como materiales de desecho de una posible oficina contable, señalando además cómo las estrategias de gestión de los residuos y el impacto de ocupaciones posteriores han podido ser determinantes en los procesos de conservación de este material. Se considera además que estaríamos ante los restos de un sistema matemático de uso extendido.

\section{LABURPENA}

El Castillon (Santa Eulalia de Tábara, Zamora) tokitik datozen arbel zati numeralen aurkikuntza aurkeztu da. Tokia gotortutako landa-kokagunea da, V-VI. mendeetan okupazioa izan zuena, eta horren gainean eraiki zen eliza bat IX-X mendeetan Arbelak elizan egindako esku-hartzean lortu ziren, baina ziurrenik erromatarren ondorengo okupazioarekin lotu beharko lirateke. Artikuluan, arbel numeral horiek aztertu dira eta kontularitzako bulego posible baten hondakin-material gisa aztertu dira; gainera, hondakinen kudeaketa-estrategiak eta ondorengo okupazioen eragina material horren kontserbazio-prozesuetan erabakigarriak izan zitezkeela adierazi da. Erabilera zabalduko sistema matematiko baten aztarnak direla uste dugu, gainera.

\section{ABSTRACT}

This paper is focused on the site of El Castillón (Santa Eulalia de Tábara, Zamora) and the numerical slates found there. It was a fortified rural settlement with a strong occupation during 5th and 6th centuries, including domestic units, metallurgical kilns, a lot of pottery sherds, some of them of high-level productions, and faunal remains. After an hiatus, the site was re-occupied in the 9th-10th centuries, when a monumentalized church linked to some burials was constructed. During the excavation of that church, two small fragments of numerical slates were found as removed or reused materials. Those slates have the same features than others from different sites. But there is a clear difference with other fortified rural settlements where great concentrations of slates have been found, probably as a result of accounting needs related to the control of tolls. Those slates were not archive documents if not a preliminary note discarded once the information was copied on a different text. The management of waste was a key to understand the archaeological secondary position of those pieces and their preservation, because an intense occupation of the site may be the cause of the destruction of the slates. The hypothesis is that El Castillón seems to have been an influential "central place" in Post-Roman times, with the use of accountability in slates, a material that came from other near areas. But the construction of the church disturbed the prior waste deposits and some of the slates were part of the building operations of the 9th-10th centuries. Another conclusion is the spread of the mathematical system seen on the slates, with could be used with very different functions, so it must not be understood as a single pattern.

\footnotetext{
(1) Departamento de Historia Medieval, Moderna y Contemporánea. Facultad de Geografía e Historia. Universidad de Salamanca. C/ Cervantes s/n 37002 Salamanca. Tfno: 923294400, ext.6250. E-mail: viso@usal.es

(2) Asociación Científico-Cultural Zamora Protohistórica
} 


\section{LAS PIZARRAS VISIGODAS Y SUS CONTEX- TOS ARQUEOLÓGICOS}

Las pizarras con inscripciones escritas configuran uno de los corpus más interesantes del periodo posromano en la península ibérica. Se trata de un conjunto muy dispar por lo que debe partirse de una tipología. Las más conocidas son las pizarras con textos escritos con contenidos muy diversos (Velázquez Soriano, 2004). Estos documentos proceden fundamentalmente del suroeste de la Meseta del Duero, con una especial concentración en el lugar de Dehesa del Castillo (Diego Álvaro, Ávila). La escasez de documentación privada para la Hispania posromana así como el hecho de que procedan de un ámbito alejado de los centros de poder, donde no existían poderosas instituciones, dotan de una enorme relevancia a este conjunto de textos (Martín Viso 2007). Otra tipología está conformada por las pizarras con dibujo, en las que se han conservado representaciones de difícil interpretación. Sin embargo, las más abundantes corresponden a un tercer patrón: las pizarras numerales. Estas se encuentran formadas por anotaciones numerales sin que se identifique aquello que se anota (Velázquez Soriano 1989: 31). El análisis de algunos ejemplares que se han conservado íntegramente ha permitido reconocer que se trataba de líneas que sumaban la misma cantidad -diferente en cada piezamediante diversas combinaciones numéricas. En una de las denominadas Tablillas Albertini, tablillas de madera referidas a una gran propiedad procedente del Norte de África en el siglo V, aparece un texto semejante (Courtois et al. 1952), por lo que cabe pensar en un sistema matemático que parece haber estado en funcionamiento en el Mediterráneo occidental.

En los últimos años, se han planteado nuevas vías de interpretación gracias a la incorporación de los datos arqueológicos, que han permitido reconocer los contextos en los que aparecen las pizarras (Martín Viso, 2013). Se han podido identificar algunas en ámbitos vilicarios que remiten al siglo IV o comienzos del siglo V, como ocurre con San Pelayo (Aldealengua, Salamanca) (Dahí Elena, 2007), Los Azafranales (Coca, Segovia) (Pérez González y Reyes Hernández, 2009: 54-56) y Valdelobos (Montijo, Badajoz) (Cordero Ruiz y Martín Viso, 2012). Las piezas, casi siempre conservadas de manera fragmentaria, se hallaban en basureros o en posición secundaria, en alguna ocasión reaprovechadas en tumbas (Cordero Ruiz y Martín Viso, 2012). A partir de estos datos, puede concluirse que el sistema numeral y el uso de la pizarra para estas anotaciones ya existía en el contexto de las uillae tardorromanas, quizás como parte de la gestión asociada a estos lugares. Por otra parte, los contextos específicos parecen indicar que era un material rápidamente amortizado.

El final de las uillae en el siglo $V$ coincide con una expansión de las pizarras con inscripciones numerales que comienzan a ser frecuentes en distintos contextos. Se hallan en algunas ciudades, como Ávila (Díaz de la Torre, 2003; Centeno Cea, 2006: 129) y Coca (Sáez Sánchez, 1985; Blanco García, 1998), pero también en algunos asentamientos rurales abiertos (Urbina Álvarez, 2006; Strato 2013; Martín Viso, 2015) y en espacios asociados a la producción (Gómez Gandullo, 2006; Paricio Alonso y Vinuesa Chao, 2009). Esta proliferación parece ser un indicio claro de la multiplicación de su uso, posiblemente con funciones muy diferentes.

Pero llama la atención su presencia en los asentamientos rurales fortificados que surgieron a partir del siglo V. Parece que estos lugares fueron creados en el marco de la desvertebración del horizonte imperial romano por iniciativa de poderes locales que se hicieron con el control efectivo de territorios de pequeña escala (Quirós Castillo, 2013; Tejerizo García, 2017: 100-109; Tejerizo García y Canosa Betés, 2018). Puede hablarse de "lugares centrales" muy heterogéneos que en el área sur del Duero no parecen relacionarse con la presencia directa de ningún tipo de autoridad centralizada. Es en estos sitios donde se detectan las mayores concentraciones de hallazgos de pizarras numerales. Así ocurre con El Cortinal de San Juan (Salvatierra de Tormes, Salamanca), La Cabeza de Navasangil (Solosancho, Ávila) y sobre todo Lerilla (Zamarra, Salamanca) (Díaz y Martín Viso, 2011; Caballero Arribas y Peñas Pedrero, 2012; Martín Viso, 2018). A pesar de haberse han encontrado fuera de posición primaria, estas piezas se han identificado con el material usado en oficinas contables situadas en estos lugares. La presencia de unas pocas pizarras con texto, asociadas a listados de antropónimos, así como la relación con representaciones de animales o de barcos evidenciarían una posible conexión entre estas pizarras y el pago de peajes locales, que habrían constituido una de las principales claves del control político asociado a los asentamientos fortificados (Martín Viso, 2013). Una situación que contrasta con el caso de los poblados campesinos abiertos, donde el número de piezas recuperadas es siempre muy bajo, por lo que no existirían oficinas contables; serían, en cambio, la huella de relaciones de poder quizás no muy continuadas (Martín Viso, 2015). La ausencia de concentraciones en las ciudades pudo obedecer simplemente a la intensa ocupación que han sufrido; de hecho, allí donde no se ha producido esa densa ocupación, como ocurre en ciertas áreas de la antigua Cauca (Coca, Segovia), se ha podido recuperar un número también significativo de piezas, que se asocian a oficinas contables (Blanco García, 1988: 55)

En general, los hallazgos de pizarras numerales se localizan preferentemente en el Suroeste de la Meseta del Duero. Sin embargo, más allá de ese foco, se han recuperado piezas en otras zonas, en especial en distintas áreas la Meseta del Duero (Represa, 1976; Mañanes Pérez, 2002: 77, 107-111 y 277-278; Regueras Grande y Martín Benito, 1997), en algunos asentamientos fortificados al otro lado del Sistema Central, como El Cancho del Confesionario (Manzanares el Real, Madrid) (Caballero Zoreda y Megías Pérez, 1977) y también en Portugal (Tente y Soares, 2007). No obstante, salvo casos 


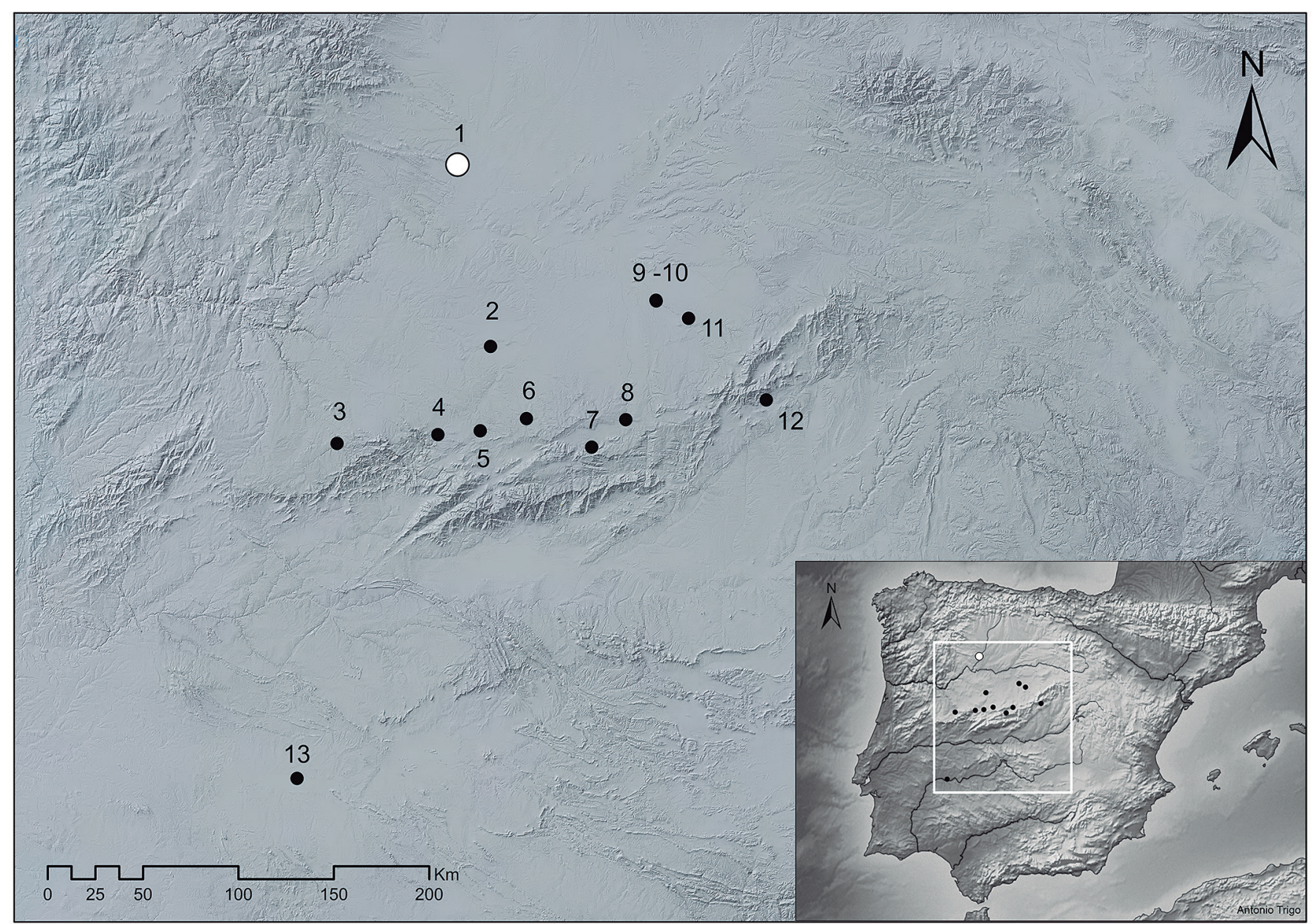

Fig.1. Lugares citados en el estudio / Sites cited on the text: 1.- El Castillón (Santa Eulalia de Tábara, Zamora); 2.- San Pelayo (Aldealengua, Salamanca): 3. Lerilla (Zamarra, Salamanca); 4.- Monte el Alcaide (Monleón, Salamanca); 5.- El Cortinal de San Juan (Salvatierra de Tormes, Salamanca); 6. - Dehesa del Castillo (Diego Álvaro, Ávila); 7.- La Cabeza de Navasangil (Solosancho, Ávila), 8.- Ávila ciudad; 9.- Los Azafranales (Coca, Segovia); 10.- Cauca (Coca, Segovia); 11.- Cerro de la Virgen del Castillo (Bernardos, Segovia); 12.- El Cancho del Confesionario (Manzanares el Real, Madrid); 13.- Valdelobos (Montijo, Badajoz).

excepcionales, la información arqueológica sigue siendo escasa. Por consiguiente, surgen preguntas sobre si el modelo explicativo, creado a partir de los datos del Suroeste de la Meseta, es aplicable a otros puntos. Para ello, es necesario que se cuente con más datos sobre contextos arqueológicos específicos. Este trabajo aporta precisamente un nuevo hallazgo y pretende ofrecer un marco de discusión a partir de la consideración de las pizarras como un material arqueológico.

\section{EL CASTILLÓN Y SUS FASES DE OCUPACIÓN}

El yacimiento de El Castillón se encuentra en la localidad de Santa Eulalia de Tábara (Zamora), situado en un espolón sobre el río Esla. Se trata de un lugar sobre el que se vienen desarrollando una serie de campañas desde el año 2007, que han permitido documentar un importante asentamiento con al menos dos fases de ocupación claramente definidas: una que correspondería a un momento posromano (siglos $\mathrm{V}$-VI) y otra que se abriría a partir de finales del siglo IX (Sastre Blanco, 2017; Sastre Blanco y Catalán Ramos, 2012; Sastre Blanco et al., 2014 y 2018).
La primera de estas fases de ocupación correspondería al periodo de mayor expansión urbanística del poblado. Aunque se han detectado evidencias de una ocupación en la Edad del Hierro, estas son residuales y quizás la profunda reordenación del área y su intensa ocupación a partir del siglo $\mathrm{V}$ supusieron un arrasamiento de las estructuras preexistentes. A pesar de la existencia de materiales cerámicos que podrían ser datados en el siglo IV, como algunos platos de TSHT, su presencia no implica una ocupación en esos momentos, ya que estos objetos pudieron tener una vida dilatada que superase el momento de su producción y porque sabemos que el final de estas producciones debió hacerse efectivo en la Meseta del Duero en torno a 430 (Vigil-Escalera Guirado, 2015: 151-155; Tejerizo García, 2017: 88-90). En esa fase se construyó una muralla perimetral que rodeaba y protegía el poblado. En su interior, se han podido identificar varios complejos habitacionales y se han estudiado aspectos sobre la vida dentro del poblado. Se observa cómo las actividades económicas en este periodo se caracterizaban por la complementariedad de las prácticas agrarias y ganaderas. Con respecto a estas últimas, se han po- 


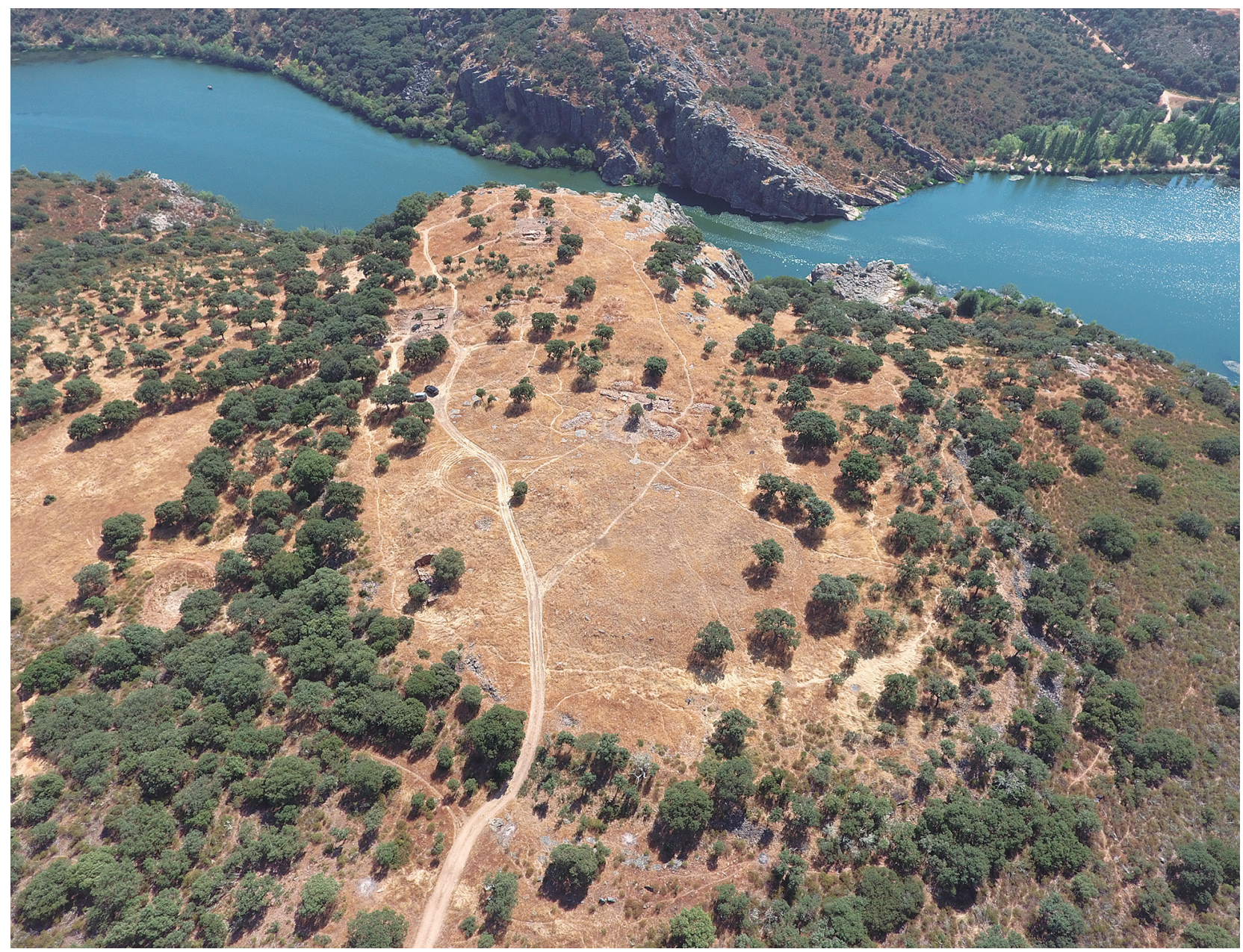

Fig.2. Localización e imagen del yacimiento de El Castillón. / The site of El Castillón and its situation.

dido atestiguar grandes acumulaciones faunísticas en espacios destinados al almacenamiento y procesado de este tipo de productos, así como la gran variedad de especies que se han documentado, procedentes tanto de actividades ganaderas, de caza y pesca (Sastre Blanco, 2017). Otro aspecto que conviene resaltar es la evidencia de hornos para uso metalúrgico. El estudio de los cerca de 93 kgs de escoria de hierro recuperados ha permitido identificar su origen en la Sierra de la Culebra, un espacio situado a unos $20 \mathrm{kms}$ del yacimiento (Sastre Blanco et al., 2011). Por otro lado, se ha recogido una gran cantidad de cerámica de este periodo, predominando las producciones locales y de cocina, que deben relacionarse con una actividad doméstica, aunque también se han recuperado cerámicas de alto nivel tecnológico, como las grises estampilladas (Sastre Blanco et al., 2018). Esta tipología se asocia a circuitos de intercambios regionales o supraregionales y su producción debe situarse entre el siglo V y los primeros decenios del siglo VI (Tejerizo García, 2018). Aunque se trate de un porcentaje reducido del total de fragmentos, su presencia revela conexiones extralocales y posiblemente desigualdades internas. Por último, y dentro de contextos habitacionales, debido a que no se han podido reconocer hasta el momento los espacios funerarios, se han podido recuperar algunas fíbulas de bronce.

A tenor de estos datos, El Castillón debe identificarse con un asentamiento rural fortificado asociado a la creación de nuevos "lugares centrales" con la desaparición del horizonte imperial romano. El Castillón proporciona una primera visión de conjunto de uno de estos asentamientos. El amurallamiento no implicaba una función meramente militar, como se ha planteado en otros casos cercanos (Nuño González y Domínguez Bolaños, 2014), sino que se observa claramente que hay una ocupación doméstica, asociada a grupos no militares. Entre los habitantes de El Castillón había diferencias sociales, plasmadas en el ámbito material a partir de la presencia de objetos como broches, cerámicas de mayor calidad e incluso una espada. Todos ellos parecen ser marcadores de estatus, al ser piezas procedentes de ámbitos extralocales, pero también por su significado cultural, a lo que se añade su carácter cuantitativamente marginal en el contexto de los materiales recuperados. La dieta de algunos de los habi- 
tantes de El Castillón, según se desprende del análisis faunístico, incorporaba aves y animales de caza, otra huella de esa desigualdad interna. Conviene resaltar la importancia adquirida por ciertas actividades artesanales, como el procesado de alimentos y muy especialmente la metalurgia, que se concentraba en un área específica y que conllevaba algún tipo de tráfico comercial o de dominio político con espacios cercanos, pero no inmediatos. En definitiva, un asentamiento de cierta densidad demográfica, con grupos de estatus y posiblemente desde el que ciertas elites ejercían un control político a partir del siglo V. Aunque el final de la ocupación no está muy bien definido, resulta bastante probable que no superase la segunda mitad del siglo VI, dada la ausencia de materiales y dataciones posteriores a esa fecha.

Después de un hiato de varios siglos en los que no se ha podido documentar una ocupación efectiva de El Castillón, el lugar vuelve a ser ocupado en el siglo IX. Los trabajos efectuados en octubre de 2018 revelaron la existencia de una edificación con dos naves bien identificadas y una posible tercera nave. Se trata posiblemente de una iglesia que estaba asociada a algunos enterramientos, En el interior de la nave central, se exhumaron tres inhumaciones infantiles que han podido ser fechadas a través de C14. Las dataciones obtenidas en el Laboratorio Beta Analytic y calibradas con el programa OxCal se sitúan en 861-988 AD (Tumba II y Tumba III) y 887-1013 AD (Tumba IV). Estos datos parecen indicar que el edificio estaba ya en uso entre finales del siglo IX y el siglo X. Debe tenerse en cuenta cómo en las inmediaciones del yacimiento se han podido encontrar algunas piezas escultóricas y epigráficas que remiten a este mismo periodo y que son un síntoma de la erección de edificaciones eclesiásticas monumentales (Regueras Grande y García-Aráez Ferrer, 2001). De hecho, existe un relato hagiográfico relacionado con la vida de san Froilán, supuesto obispo de León a finales del siglo IX y comienzos del siglo $X$, en el que se menciona cómo este personaje fundó un monasterio en un lugar alto junto al río Esla (Martín Iglesias, 2009: 582). Puede plantearse como hipótesis que esa cita se refiera a la iglesia de El Castillón, emplazada precisamente en un lugar que responde a la descripción del texto.

Más allá de esa presunta identificación, los datos arqueológicos revelan una inversión monumental, que coincide con la afirmación de la autoridad asturiana sobre la Meseta del Duero. Sin embargo, los estudios arqueológicos sobre estos iglesias y monasterios monumentales surgidos a finales del siglo IX en la región duriense son prácticamente inexistentes, con la salvedad del caso de San Miguel de Escalada (Larrén Izquierdo y Campomanes Alvarado, 2014). El Castillón ofrece, por tanto, la posibilidad de estudiar desde el punto de vista material este tipo de construcción. La elección de un antiguo asentamiento fortificado, que en esos momentos no estaba ocupado, no debió ser casual sino que tuvo que obedecer a pautas relacionadas con la legitimación de las nuevas formas de dominio implementadas a partir de esos momentos.

\section{EL HALLAZGO DE PIZARRAS}

Se han podido documentar dos fragmentos de pizarras numerales en ambas naves laterales de la posible iglesia. La primera de las piezas, se localizó en la Estancia 01 de este edificio, identificada con la nave lateral oriental, en un nivel de espolio antiguo efectuado (UE: 11.075), lo que había provocado la destrucción del nivel de circulación hasta llegar al nivel geológico. El objetivo de esta destrucción intencionada pudo ser la extracción de materiales de construcción asociados al edificio o de algún otro tipo de elemento que se encontrase allí en el último momento de abandono de este lugar. Tampoco se puede desechar la posibilidad de espolio de alguna tumba, como se documenta en algún otro caso (Tumba III). Es interesante advertir que el fragmento de pizarra numeral se encontraba junto a otros materiales muy relevantes, como una variedad de cerámicas medievales y tardoantiguas, un fragmento correspondiente a una espada de hierro, una cuenta de vidrio gallonada, y una moneda de Enrique II (1369-1379). Se trata de materiales muy variados, producto del espolio acontecido en este lugar que ha mezclado materiales procedentes de diferentes niveles estratigráficos.

El segundo fragmento de pizarra numeral se localizó en la nave lateral occidental, cerca del espacio de acceso entre esta nave y la central que permitiría la comunicación entre ambas estancias. Se encontraba en un nivel de espolio (UE: 11.092) efectuado junto a la entrada de lateral de la nave central, en una zona que se encontraba cubierta por una especie de opus signinum de coloración rojiza. Este estrato presentaba una destrucción con la finalidad de retirar de esta zona algún tipo de elemento o material constructivo asociado a este conjunto religioso, o asociado a los enterramientos que se localizan en este sector. Al igual que sucede en el caso anterior nos encontramos con una mezcla de materiales procedentes de diferentes niveles a causa del espolio efectuado en la zona y, en concreto, con fragmentos de TSHT.

La primera de las piezas (UE: 11.075) es un fragmento de pequeñas dimensiones $(65 \times 37 \mathrm{mms}$, un grosor de 7-7,5 mms y un peso de 30,62 grs). Se trata de una pieza de coloración marrón pardo y la superficie de la pizarra donde se ha realizado la escritura parece haber recibido un tratamiento previo, a través de un pulido de la superficie para realizar después la escritura en dicha cara. La escritura se ha realizado mediante un trazo grueso y profundo, realizado probablemente con algún tipo de punzón o elemento metálico. Presenta cuatro fragmentos de líneas de escritura, aunque tanto la primera como la última están fracturadas lo que hace que los signos se hallen incompletos. No obstante, se puede observar la presencia de signos como I (en una ocasión aparece unido como II) y V, lo que corresponde 


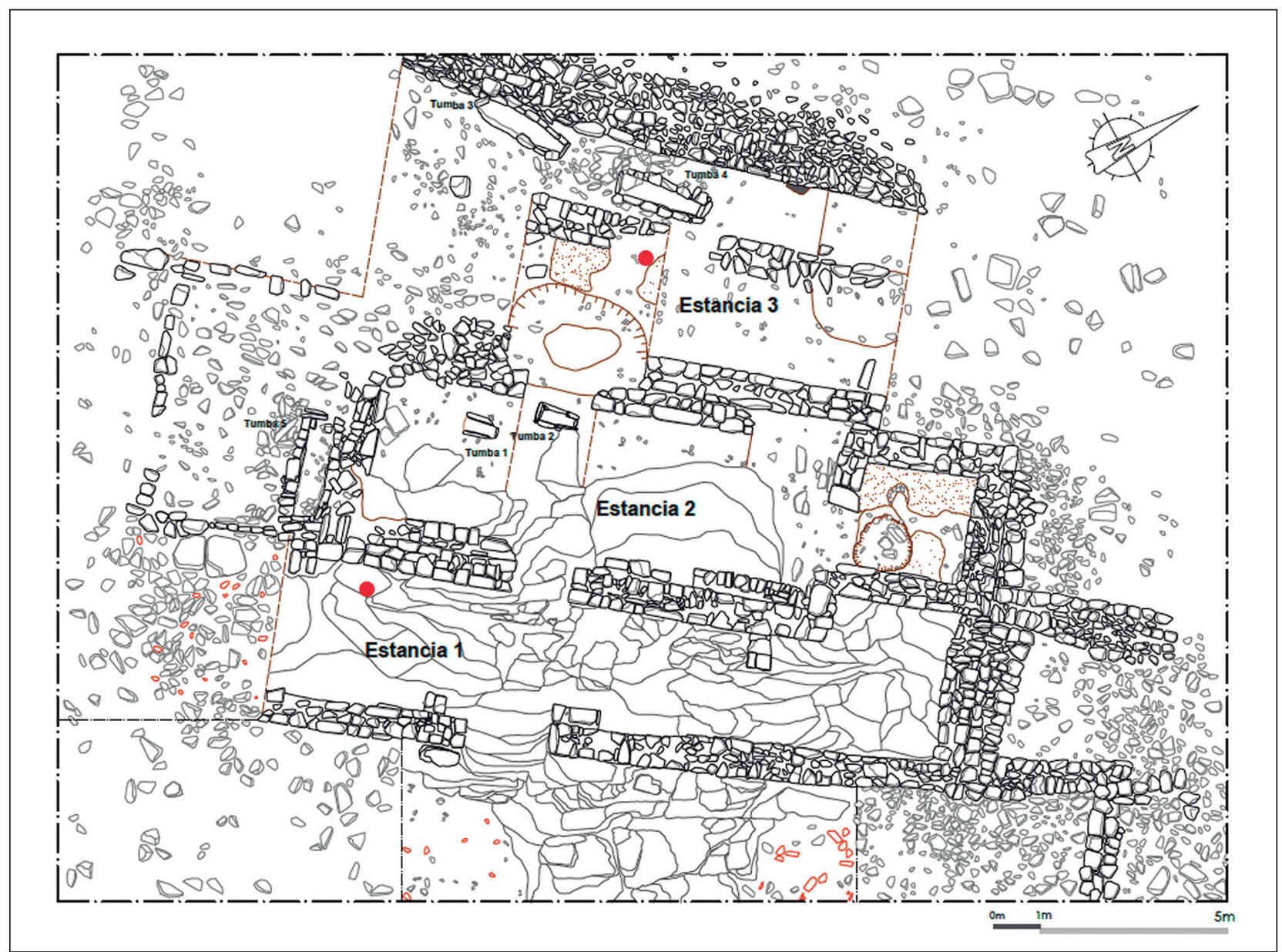

Fig.3. Plano de la excavación de la iglesia de El Castillón, con indicación de los lugares donde se hallaron las pizarras. / Plan of the excavation of the church of El Castillón, with indication of the places where the slates were found.

con el patrón más habitual en el que los números más altos son muy infrecuentes. No se detectan renglones que dividan las distintas líneas y está escrita tan solo por una de las caras. La transcripción sería la siguiente:

\section{| | | |}

\section{$\mathrm{V}|\mathrm{I}|$}

\section{| || || |}

El segundo fragmento (UE: 11.092) es una pieza cuadrangular, (46 x $42 \mathrm{mms}$, un grosor de 3-4 mms y un peso de $12,56 \mathrm{gr}$ ). Se trata de una pizarra de color negro sin ningún tipo de tratamiento en la superficie. Se compone de tres líneas que presentan un menor cuidado con respecto a la otra pieza, con renglones torcidos, y escrita igualmente por una sola línea, con un trazado más fino y menos profundo. La primera de las líneas conserva los números parcialmente, por lo que se ofrece una reconstrucción hipotética. En cuanto a las cifras empleadas, aparecen sobre todo I, pero llama la atención la presencia de una $\mathrm{X}$, un tanto descuidada, al final de la segunda línea. Aunque se ha optado por leerla como parte de las cifras incisas, no es descartable que se trate del efecto de una marca posterior provocada por cualquier tipo de utensilio. De igual manera, el signo más a la izquierda de esa misma línea resulta confuso, pero podría leerse como una $\mathrm{V}$, aunque escrita de forma muy abierta. A pesar de mostrar más variedad en cuanto a los signos que el primero de los fragmentos, de nuevo nos hallamos ante un patrón frecuente con combinaciones de I, V y X. La transcripción sería la siguiente:

I | |

$\vee \pi x$

III III।

Por tanto, se trata de dos fragmentos de dos piezas distintas. Resulta imposible reconstruir las líneas completas ni tampoco es factible identificar qué era lo que se estaba contando en esas piezas. Una situación frecuente, ya que, salvo algunos dibujos en algunas piezas que podrían evocar posibles tasas sobre el tráfico fluvial o el ganado (Díaz y Martín Viso, 2011; Martín Viso, 2018), en general no hay indicios que permitan conjeturar el objeto de esta contabilidad. 


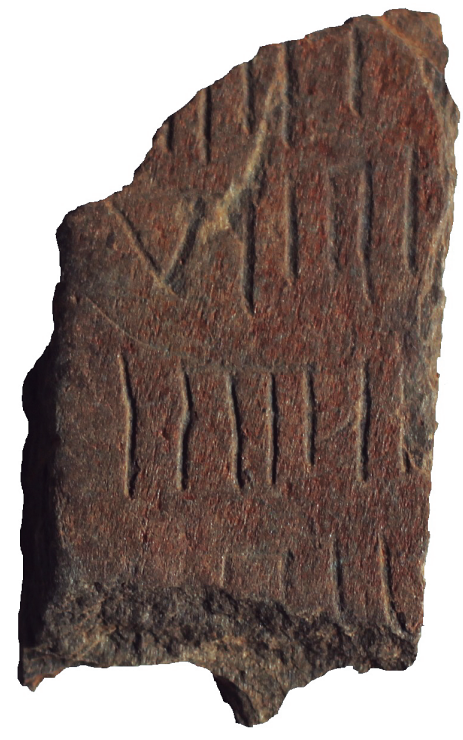

Fig.4. Pizarra de El Castillón (UE 11.075). / Slate from El Castillón (UE 11.075)

Las pizarras y los esquistos son elementos líticos presentes y abundantes en el yacimiento en todo tipo de materiales, incluidos los constructivos. Sin embargo, el terreno en el que se encuentra el yacimiento así como su entorno más próximo se compone fundamentalmente de cuarcitas. Las pizarras son el material predominante en la Sierra de la Culebra y en la Tierra de Aliste, espacios comarcales cercanos, pero no inmediatos. Por tanto, y al igual que sucediera en el caso del hierro, parece existir una conexión entre este "núcleo central" y unas áreas cercanas con las que había algún tipo de flujo al menos económico. No es una situación extraña, porque en otros casos también se ha puesto de manifiesto cómo se estaba usando un material que no era local (Cordero Ruiz y Martín Viso, 2012).

Con respecto a la cronología de estas piezas, el contexto arqueológico no es claro, debido a la construcción de la iglesia a finales del siglo IX o principios del X. Sin embargo, puede plantearse como hipótesis que se trate de materiales procedentes de algún momento de la fase posromana (siglos V-VI). Los fragmentos fueron reutilizados como parte del movimiento de tierras originado por la edificación de la iglesia o como material asociado a la construcción de tumbas. En ambos casos, se trataría de posiciones secundarias de elementos procedentes de una fase previa a la construcción de la iglesia. Por otro lado, la cronología de esas piezas en el conjunto de la Península Ibérica coincide con esa datación y las escasas evidencias de pizarras inscritas de los siglos VIII y IX se corresponden con textos escritos de tipo filacteria (Esparza Arroyo y Martín Valls, 1998; Velázquez Soriano, 2004; Velázquez Soriano, 2018).
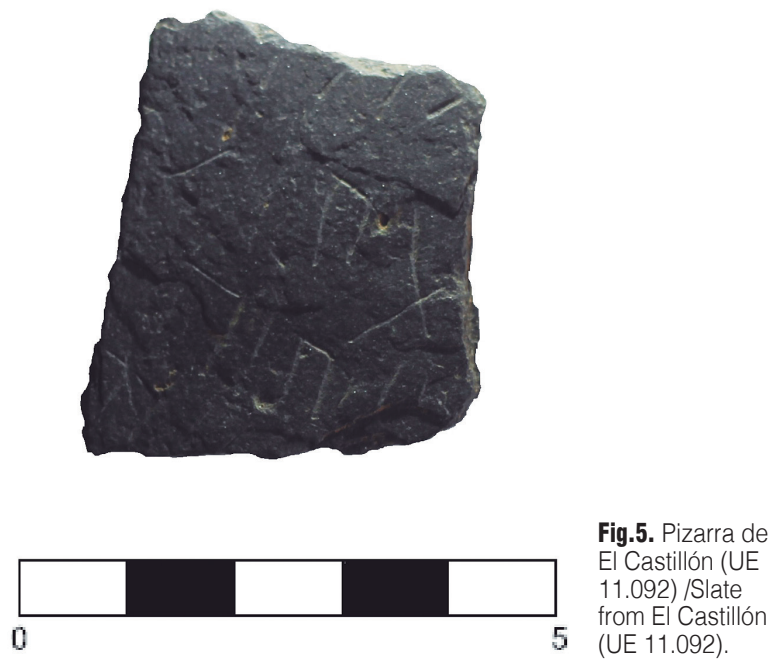

\section{PIZARRAS, OFICINAS CONTABLES Y GES- TIÓN DE LOS RESIDUOS}

El hallazgo de estos dos nuevos fragmentos de pizarra abre algunas puertas para el análisis de un tipo de material elusivo y complejo en cuanto a su interpretación. Un primer aspecto que debe resaltarse es que el patrón de uso de las pizarras numerales tiene una gran extensión geográfica. Por tanto, parece que la utilización de este tipo de herramientas contables estaba relativamente generalizada. Es probable que el empleo de otros soportes, menos resistentes, haya provocado una desaparición de buena parte de la evidencia. La elección de la pizarra como soporte ha permitido, en cambio, la pervivencia de ejemplos de ese uso (Martín Viso, 2013). Es interesante advertir que, junto a zonas donde este tipo de rocas son abundantes, también se han hallado ejemplares en áreas en cuyas cercanías no existía esa fácil obtención. Así sucede en El Castillón, por lo que puede afirmarse que había algún tipo de intercambio que incluía la pizarra, reconocida como un posible soporte para usos escriturísticos. En cuanto al tipo de herramienta contable, los fragmentos hallados en El Castillón no arrojan nuevos datos con respecto a lo que hasta ahora se había planteado. En todo caso, parece que se trataba de un sistema bastante generalizado, cuya visibilidad es escasa ya que debía ser un tipo de documentación que no estaba destinada a la conservación. Esa generalización aboga por una multiplicidad de posibles funciones, pero también puede entenderse como una huella de la existencia de un saber matemático relativamente extendido por la Península Ibérica y el Mediterráneo Occidental.

Los fragmentos hallados en El Castillón se hallaban en un contexto secundario. En aquellos casos donde se ha podido documentar arqueológicamente el hallazgo de pizarras numerales, esta situación se repite (Gutiérrez Palacios, Díaz y Díaz y Maluquer de Motes, 1958; Storch de Gracia, 1998: 151-154; Urbina Álvarez, 
2002; Centeno Cea, 2006; Dahí Elena, 2007; Tente y Soares, 2007; Cordero Ruiz y Martín Viso, 2012; Strato, 2013; Martín Viso, 2013; Martín Viso, 2015). La única excepción parece ser Monte el Alcaide (Monleón) donde aparecieron tres grandes pizarras numerales colocadas horizontalmente y relacionadas con una pileta de granito abierto, con una capacidad de unos 28 litros. En este caso, parece que puede inferirse una relación entre las pizarras y los líquidos que allí se medían (vino o más probablemente aceite). Esa conexión parece reforzarse por el carácter unifásico del yacimiento, que se abandonó tras un corto periodo de uso (Paricio Alonso y Vinuesa Chao, 2009). Puede pensarse en una contabilidad inmediata, con unas inscripciones que estaban allí depositadas. Pero no formaban una suerte de archivo y quizás un abandono rápido propició la conservación de ese depósito, mientras otros lugares tuvieron una ocupación más dilatada e intensiva que conllevó una amortización más completa de esos materiales. En el caso de que fueran una contabilidad inmediata que no estaba destinada a ser archivada, una vez que dejaban de ser útiles -posiblemente con la elaboración de otros documentos más expresivos-, se trataban como un desecho y podían ser reutilizadas como material constructivo o permanecer, en forma de pequeños fragmentos, en basureros o simplemente en el piso del sitio. Los datos de El Castillón parecen avalar esa situación. Debían formar parte de materiales de desecho, quizás removidos en la obra de la iglesia de los siglos IX-X o reusados en las tumbas. No había, por tanto, un archivo de estas pizarras.

Por otro lado, El Castillón fue un asentamiento rural fortificado, un tipo de yacimientos que ha sido frecuentemente asociado a la presencia de pizarras numerales. Frente a la acumulación de numerosas piezas que se detecta en otros ejemplos (Lerilla, El Cortinal de San Juan, La Cabeza de Navasangil), consecuencia de la existencia de oficinas contables (Díaz y Martín Viso, 2011), el número de fragmentos en El Castillón es muy exiguo. No se trata de una excepción, ya que esta misma realidad se repite en el Cerro de la Virgen del Castillo (Bernardos, Segovia) (Urbina Álvarez, 2002), mientras que en otras ocasiones no disponemos de una información exacta sobre el número de piezas recuperadas, como en El Cancho del Confesionario (Caballero Zoreda y Megías Pérez, 1977). Podría interpretarse este hecho como la inexistencia de fenómenos de concentración de pizarras y, por consiguiente, la ausencia de oficinas contables. Ahora bien, una diferencia clave entre asentamientos rurales fortificados con acumulación de pizarras numerales y aquellos donde no se ha podido comprobar estriba en la presencia o no de ocupaciones posteriores. En Lerilla o El Cortinal de San Juan no se ha podido detectar una ocupación a la fase posromana, mientras en La Cabeza de Navasangil pudo darse una poco intensa fase plenomedieval (Tejerizo García y Vigil-Escalera, 2017: 148). En cambio, en Virgen del Castillo (Bernardos) se observa una importante intervención posterior que implicó reformas en la muralla (Gonzalo Gonzalo, 2006: 91). En El Castillón, la construcción de la iglesia también supuso una fase nueva de ordenación. Es factible pensar que estas situaciones pudieron alterar depósitos previos, por ejemplo mediante la remoción o limpieza de áreas de desecho, que tuvieron como resultado la desaparición de las evidencias de una posible concentración en forma de basureros de una oficina contable. En cualquier caso, llama la atención cómo en El Castillón las pizarras no han aparecido en las estructuras de los siglos V-VI que han sido exhumadas. Quizás no sea allí donde deban encontrarse, sino en torno a otros espacios del sitio, ya que los hallazgos de pizarras dentro de los yacimientos suelen concentrarse en puntos muy concretos, posiblemente en el lugar donde se encontraban las oficinas contables, como sucede en El Cortinal de San Juan (Díaz y Martín Viso, 2011). En El Castillón, se han podido documentar espacios de vertedero con grandes cantidades de restos faunísticos, que también se detectan en zonas de almacenamiento en el centro del poblado (Sondeo 3). En cambio, no se han recuperado pizarras en tales espacios, lo que probablemente incide en que las actividades relacionadas con la contabilidad reflejada en esas piezas se situaban en otros puntos y que el patrón de gestión de los desechos de esas oficinas contables estaba diferenciado. Una hipótesis factible es que la oficina contable posromana se hallase en el sector afectado por la construcción de la iglesia, lo que explicaría su presencia entre los materiales removidos.

En cualquier caso, resulta interesante subrayar la importancia de las actividades económicas documentadas en la primera fase de ocupación de El Castillón. Junto con actividades agrarias -se ha recogido una amplia muestra de semillas en fase de análisis- y con las asociadas a los usos de la fauna, es relevante el papel de la producción metalúrgica. No sabemos si alguna de estas producciones pudo ser el objetivo de una contabilidad que apenas vislumbramos. Pudieron haberse registrado o bien materias primas que llegaban al lugar en forma de intercambio o de pago obligado, o bien productos ya elaborados. En cualquier caso, la conexión con la Sierra de la Culebra parece haber sido bastante estrecha. Cabe preguntarse hasta qué punto esa relación pudo tener una base política y ser la consecuencia del dominio ejercido desde este punto sobre el entorno territorial, habida cuenta de la ausencia de asentamientos rurales fortificados de este periodo en la Sierra de la Culebra.

Por supuesto, esta realidad coexistió con un uso más amplio de la pizarra como soporte para la reproducción de este sistema matemático. La conclusión es de nuevo que estaríamos ante un patrón cultural ampliamente conocido, del que solo se han conservado algunos fragmentos cuando estos se inscribieron en un material pétreo con una amplia perduración. Su presencia en ámbitos aldeanos sigue las mismas pautas, 
ya que se encuentran en posición secundaria, por lo que su tiempo de uso debió ser muy corto. Ahora bien, en estos asentamientos abiertos es interesante advertir la ausencia de concentración: se hallan unos pocos ejemplares, en general muy fragmentados. Quizás esto se deba a que la gestión de los desechos en estos lugares, lo que habría provocado la desaparición de los residuos emanados de la contabilidad. Pero parece razonable pensar que se trate de un hecho vinculado a que no había en estos sitios esas oficinas contables: o bien eran la evidencia de un control muy esporádico y puntual sobre algún tipo de producción o bien mostrarían que el sistema estaba difundido y también se usaba para otras funciones en un ámbito doméstico. El dato de que las únicas concentraciones de pizarras numerales se encuentren en asentamientos rurales fortificados sería un indicio de que al menos allí existían esas oficinas que precisaban de una contabilidad constante de bienes que estaban a la vista.

\section{CONCLUSIÓN}

El análisis de los fragmentos de pizarras numerales hallados en El Castillón representa una huella de la generalización de un sistema matemático ampliamente difundido en los primeros siglos posromanos en la Península Ibérica y posiblemente en amplias zonas del Mediterráneo occidental. Es muy probable que sus orígenes se encuentren en momentos previos, a tenor de la reutilización de algunas pizarras en contextos tardorromanos. Sin embargo, su punto de máxima difusión coincidió con la desarticulación del sistema romano y quizás fue un efecto de la traslación a nuevos ámbitos de prácticas reservadas hasta entonces a entornos vilicarios. En cualquier caso, este sistema no se relacionaba exclusivamente con una sola funcionalidad, sino que los usos debieron ser muy diversos.

En el caso de El Castillón, nos encontramos con un asentamiento rural fortificado, uno de los escenarios más frecuentes de los hallazgos de este tipo de piezas, mientras que las relacionadas con textos escritos responden a otros patrones. Como en otras ocasiones, se han encontrado en posición secundaria, dentro de las estructuras asociadas a la fase de ocupación que se iniciaría a finales del siglo IX y comienzos del X con la construcción de una iglesia. Por tanto, su cronología debe situarse en la fase de ocupación previa, de los siglos V-VI, lo que coincide con las cronologías habituales en este tipo de piezas. Es posible relacionar la presencia de estas pizarras con algún tipo de contabilidad. La importancia de las actividades económicas radicadas en este sitio y la ausencia de pizarras en el área inmediata podrían ser indicios de una función asociada al control de bienes que llegaban a este lugar, convertido en un centro político de escala local. El vínculo con la cercana Sierra de la Culebra podría ser consecuencia de un control de carácter político sobre ese territorio. Sin embargo, se trata de apenas dos fragmentos de dos piezas distintas, una circunstancia muy distinta a la concentración que se detecta en otras ocasiones. A pesar de ello, y teniendo siempre en cuenta que se han encontrado en posición secundaria, no se puede desechar la idea de que fueran los residuos de una oficina contable. La ausencia de otros hallazgos semejantes quizás pueda entenderse a través de los patrones de gestión de los desechos en tales lugares. Una vez usadas y desfuncionalizadas, las piezas se concentraban cerca de estas, lo que explica el patrón de hallazgos en lugares como El Cortinal de San Juan o La Cabeza de Navasangil. Sin embargo, la presencia de nuevas e intensas ocupaciones posteriores pudo alterar esos depósitos; la construcción de la iglesia altomedieval de El Castillón pudo haber producido ese efecto y únicamente se han recuperado fragmentos relacionados con la remoción de tierras.. Por tanto,los mecanismos de gestión de los residuos y la presencia de una ocupación posterior habrían sido factores clave para que no se haya recogido un mayor número de piezas, frente a lo que sucede en otros sitios semejantes. Una situación que acerca a El Castillón con los procesos que se observan en ámbitos urbanos donde hubo una intensa ocupación, como Ávila, lo que a su vez nos permite ver las similitudes entre ciudades y asentamientos rurales fortificados en áreas del centro peninsular.

Esta explicación sirve para comprender el papel de los asentamientos rurales fortificados como centros políticos y económicos de escala comarcal. En tal sentido, la presencia de pizarras implica la conexión con espacios en los que se podía obtener ese material y, en consecuencia, con redes de intercambio que podían haber sido creadas como consecuencia del dominio ejercido desde esos puntos. Pero la generalización del sistema conllevó una acusada diversidad de situaciones. El marco interpretativo de las pizarras encontradas en asentamientos rurales abiertos es muy distinto, relacionándose o bien con la presencia puntual de poderes sobre los habitantes de esos lugares o bien con una actividad de escala local. No obstante, en ambos casos los mecanismos de gestión de los residuos debieron ser muy semejantes con una rápida amortización de un material que tenía una función inmediata. Se trataba de un tipo de piezas que no se destinaban para su archivo, un rasgo que las diferenciaba de las pizarras con textos escritos, por otro lado infrecuentes en los asentamientos rurales fortificados. Aunque su soporte fuera el mismo, su uso y significado eran muy distintos.

\section{BIBLIOGRAFÍA}

Blanco García, J. F., 1988. Coca arqueológica. Revista de Arqueología 81, 46-55.

Blanco García, J. F., 1998. Aproximación a la Cauca del bajo imperio. In: Congreso internacional la Hispania de Teodosio, vol. II, 377-393. Salamanca, Junta de Castilla y León.

Caballero Arribas, J., Peñas Pedrero, D., 2012. Un castrum de época visigoda en el Valle Amblés: La Cabeza de Navasangil (Solosancho, Ávila). In: Quirós Castillo, J. A., Tejado 
Sebastián, J. M. (eds.), Los castillos medievales en el norte peninsular a través de la arqueología, 213-238. Universidad del País Vasco, Bilbao.

Caballero Zoreda, L., Megías Pérez, G., 1977. Informe de las excavaciones del poblado medieval del Cancho del Confesionario, Manzanares el Real (Madrid) Julio, 1973. Noticiario Arqueológico Hispánico 5, 325-331.

Centeno Cea, I., 2006. La ciudad entre los siglos IV y VII. EI mundo tardoantiguo. In: Estremera Portela, S. (coord.), Arqueología urbana en Ávila. La intervención en los solares del palacio de don Gaspar del Águila y Bracamonte (antiguo convento de los Padres Paúles), 115-137. Junta de Castilla y León, Valladolid.

Cordero Ruiz, T., Martín Viso, I., 2012. Sobre los usos y la cronología de las pizarras numerales. Reflexiones a partir del caso del yacimiento de Valdelobos (Montijo, Badajoz). Archivo Español de Arqueología 85, 253-266.

Courtois, C., Leschi, L., Perrat C., Saumagne. C., 1952. Tablettes Albertini. Actes privés de l'époque vandale (fin du Ve siècle). Arts et Métiers Graphiques, París.

Dahí Elena, S., 2007. Un contexto cerámico de la Antigüedad tardía: el yacimiento de San Pelayo (Aldealengua, Salamanca). Nuevos datos sobre la cronología de las pizarras visigodas. Pyrenae 38(1), 79-104.

Díaz, P. C., Martín Viso, I., 2011. Una contabilidad esquiva: las pizarras numerales visigodas y el caso de El Cortinal de San Juan (Salvatierra de Tormes, España). In: Díaz, P. C., Martín Viso, I. (eds.), Between taxation and rent. Fiscal problems from Late Antiquity to Early Middle Ages, 221-250. Edipuglia, Bari.

Díaz de la Torre, J., 2003. Informe final ( $1^{a}$ y $2^{a}$ fase) de los trabajos arqueológicos efectuados en el entorno del Episcopio (Ávila de los Caballeros). Ávila, Castellum S. Coop. [Informe inédito].

Esparza Arroyo, Á., Martín Valls, R., 1998. La pizarra altomedieval de Fuente Encalada (Zamora): contribución al estudio de las inscripciones profilácticas. Zephyrus 51, 237-262.

Gómez Gandullo, J. A., 2006. Avance de las excavaciones arqueológicas en el yacimiento de época visigoda de La Legoriza, San Martín del Castañar (Salamanca). In: Morín de Pablos, J. (ed.), La investigación arqueológica de la época visigoda en la comunidad de Madrid. Zona Arqueológica 8, vol. I, 217-235. Museo Arqueológico Regional, Alcalá de Henares.

Gonzalo Gonzalo, J. M., 2006. El Cerro del Castillo, Bernardos (Segovia). Un yacimiento singular en la provincia de Segovia durante la Antigüedad Tardía. Caja Segovia, Segovia.

Gutiérrez Palacios, A., Díaz, Díaz, M. C., Maluquer de Motes, J., 1958. Excavaciones en la Lancha del Trigo, Diego Álvaro (Ávila). Zephyrus IX, 59-78.

Larrén Izquierdo, H., Campomanes Alvaredo, E., 2014. San Miguel de Escalada a través de su arqueología: valoración de sus trabajos (1983-2004). In: García Lobo, V., Cabero Domínguez, G. (eds.), San Miguel de Escalada (913-2013), 85-122. Universidad de León, León.

Mañanes Pérez, T., 2002. Arqueología del área central de la cuenca del río Duero de Simancas a Coca. Diputación de VaIladolid, Valladolid.

Martín Iglesias, J. C., 2009. La Vita Froilanis episcopi Legionensis (BHL 3180) (s. X): introducción, edición crítica y particularidades lingüísticas. In: Goullet, M. (ed.), Parva pro magnis munera. Études de littérature tardo-antique et médiévale offertés â François Dolbeau per ses élèves, 561-584. Brepols, Turnhout.

Martín Viso, I., 2007. La sociedad rural en el suroeste de la meseta del Duero (siglos VI-VII). In: Del Ser Quijano, G., Martín Viso, I. (eds.), Espacios de poder y formas sociales en la Edad Media. Estudios dedicados a Ángel Barrios, 171-188. Universidad de Salamanca, Salamanca.

Martín Viso, I., 2013. The Visigothic Slates and their archaeological contexts. Journal of Medieval Iberian Studies 5(2), 145-168.

Martín Viso, I., 2015. Huellas del poder: pizarras y poblados campesinos en el centro de la península ibérica (siglos V-VII). Medievalismo 25, 285-314.

Martín Viso, I., 2018. Las pizarras de Lerilla. In: Fortificaciones, pizarras y poblados. La Raya en el Medievo, 314-329. Ayuntamiento de Ciudad Rodrigo, Ciudad Rodrigo.

Nuño González, J., Domínguez Bolaños, A., 2014. La muralla tardoantigua de Muelas del Pan (Zamora). Una construcción de urgencia en un tiempo convulso. In: Catalán, R., Fuentes, P., Sastre, J. C. (eds.), Las fortificaciones en la tardoantigüedad. Élites y articulación del territorio (siglos V-VIII d.C.), 297328. Madrid, La Ergástula.

Paricio Alonso, F., Vinuesa Chao, M., 2009. Monte el Alcaide: un yacimiento altomedieval en el alto valle del Alagón (Monleón, Salamanca). In: Martín Viso, I. (ed.), ¿Tiempos oscuros? Territorio y sociedad en el centro de la Península Ibérica (siglos VII-X), 45-67. Sílex, Madrid.

Pérez González, C., Reyes Hernández, O., 2009. Epigrafía caucense (II). Oppidum 5, 39-64.

Quirós Castillo, J. A., 2013. Defensive sites of the Early Middle Ages in North-West Spain. In: Baker, J., Brookes, S., Reynolds, A. (eds.), Landscapes of defence in early medieval Europe, 301-339. Brepols, Turnhout.

Regueras Grande, F., Martín Benito, J. I., 1997. Hallazgos arqueológicos en el área de Benavente. Brigecio 7, 310-311.

Regueras Grande, F., García-Aráez Ferrer, H. (eds.), 2001. Scriptorium. Tábara visigoda y mozárabe. Centro de Estudios Benaventanos Ledo del Pozo, Benavente.

Represa, M. F., 1976. Una pizarra visigótica del tipo Lerilla. Boletín del Seminario de Estudios de Arte y Arqueología XLII, 452-457.

Sáez Sánchez, C., 1985. Hallazgo de pizarras visigodas cifradas en Coca de la Vega. Anuario de Estudios Medievales 15, 35-37.

Sastre Blanco, J. C., 2017. El poblado de El Castillón (Santa Eulalia de Tábara, Zamora): contribuciones al estudio de la antigüedad tardía en el valle del Esla. Universidad de Granada, Granada (Tesis doctoral inédita).

Sastre Blanco, J. C., Criado Portal, A. J., Fuentes Melgar, P., 2011. Metalurgia del hierro en el yacimiento tardoantiguo de El Castillón (Santa Eulalia de Tábara, Zamora). In: $1^{\circ}$ Congresso Internacional, Povoamento e Exploração de Recursos Mineiros, 483 - 500. Braga.

Sastre Blanco, J. C., Catalán Ramos, R., 2012. Un asentamiento fortificado en la tardoantigüedad: el castro de El Castillón (Santa Eulalia de Tábara, Zamora). In: Quirós Castillo, J. A., Tejado Sebastián, J. M. (eds.), Los castillos altomedievales en el Noroeste de la Península Ibérica, 193-212. Universidad del País Vasco, Bilbao. 
Sastre Blanco, J. C., Catalán Ramos, R., Fuentes Melgar, P., 2014. El conjunto cerámico de El Castillón (Zamora) y las cerámicas de imitación de Sigillata en el contexto del Siglo V. In: Morais, R., Fernández A., Sousa, M.J. (eds.), As produções cerâmicas de imitação na Hispânia, vol. I, 471-481. Sociedad de Estudios de la Cerámica Antigua en España, Madrid.

Sastre Blanco, J. C., Catalán Ramos, R., Fuentes Melgar, P., Vázquez Fadón, M., Rodríguez Monterrubio, Ó., Álvarez Rodríguez, A., 2018. Producciones cerámicas en el poblado de El Castillón entre los siglos V-VI d.C. In: Martín Viso, I., Fuentes Melgar, P., Sastre Blanco, J. C., Catalán Ramos, R. (coords.), Cerámicas altomedievales en Hispania y su entorno (siglos V-VIII d.C.), 379-400. Glyphos, Valladolid.

Storch de Gracia, J. J., 1998. Avance de las primeras actividades arqueológicas en los hispano-visigodos de la Dehesa del Cañal (Pelayos, Salamanca). Arqueología, Paleontología y Etnografía 4, 141-160.

Strato, 2013. La Cárcava de la Peladera. In: Quirós Castillo, J. A. (ed.), El poblamiento rural de época visigoda en Hispania. Arqueología del campesinado en el interior peninsular, 101116. Universidad del País Vasco, Bilbao.

Tejerizo García, C., 2017. Arqueología de las sociedades campesinas en la cuenca del Duero durante la Primera Edad Media. Universidad del País Vasco, Bilbao.

Tejerizo García, C., 2018. Estampas del pasado. Un análisis contextual del material estampillado en el mundo rural de la Meseta Norte (siglos V-VI d.C.). In: Martín Viso, I., Fuentes Melgar, P., Sastre Blanco, J. C., Catalán Ramos, R. (coords.), Cerámicas altomedievales en Hispania y su entorno (siglos V-VIII d.C.), 261-283. Glyphos, Valladolid.

Tejerizo García, C., Canosa Betés, J., 2018. Power, control and social agency in post-Roman northern Iberia: an archaeological analysis of hillfort occupation. Journal of Medieval Iberian Studies 10(3), 295-323.
Tejerizo García, C., Vigil-Escalera Guirado, A. 2017. Castro Ventosa y La Cabeza de Navasangil: una revisión de sus secuencias de ocupación y el fenómeno de los asentamientos fortificados altomedievales. Nailos. Estudios Interdisciplinares de Arqueología 4, 126-161.

Tente, C., Soares, A. M., 2007. Uma pizarra visigoda com inscrição numérica em Santa Margarida (Serpa). Arqueologia Medieval 10, 13-19.

Urbina Álvarez, A., 2002. Hallazgo de dos pizarras con inscripción en el hábitat tardoantiguo del Cerro de la Virgen del Castillo (Bernardos, Segovia). Faventia 24(1), 135-144.

Urbina Álvarez, A., 2006. Pizarras con inscripción de época hispanovisigoda en la provincia de Madrid. In: Morín de Pablos, J. (ed.), La investigación arqueológica de la época visigoda en la comunidad de Madrid. Zona arqueológica 8, vol. III, 781-786. Museo Arqueológico Regional, Alcalá de Henares.

Velázquez Soriano, I., 1989. Las pizarras visigodas: edición crítica y estudio. Universidad de Murcia, Murcia.

Velázquez Soriano, I., 2004. Las pizarras visigodas (Entre el latín y su disgregación. La lengua hablada en Hispania, siglos VI-VIII). Instituto Castellano y Leonés de la Lengua, Burgos.

Velázquez Soriano, I., 2018. Pizarra opistógrafa de Crestelos. In: Fortificaciones, pizarras y poblados. La Raya en el Medievo, 363-364. Ayuntamiento de Ciudad Rodrigo, Ciudad Rodrigo.

Vigil-Escalera Guirado, A., 2015. Los primeros paisajes altomedievales en el interior de Hispania. Registros campesinos del siglo quinto a.C. Universidad del País Vasco, Bilbao. 
